\title{
MACROINVERTEBRADOS EN LAS LAGUNAS DE PUERTO VIEJO, LIMA - PERÚ
} José Iannacone $^{1}$, Jaime Mansilla ${ }^{2}$ y Karen Ventura ${ }^{3}$

\begin{abstract}
Resumen
Se realizó un estudio para evaluar la biodiversidad y similaridad de los macroinvertebrados del bentos de las lagunas de Puerto Viejo del 15 de Julio al 15 de Octubre del 2001. Se realizaron siete muestreos (M) temporales en ocho estaciones (E) espaciales en sus lagunas naturales y artificiales. Se calculó la diversidad alfa usando el Indice de Diversidad de Shannon-Wienner $\left(\mathrm{H}^{\prime}\right)$, el de Equidad de Pielou ( $\mathrm{J}^{\prime}$ ) y el Indice de Dominancia de Simpson (C). Para la determinación de la diversidad beta se empleó los Indices cuantitativos de Sörensen y Morisita-Horn. Un total de 5519 especímenes, tres filas, seis órdenes, nueve familias y 10 especies fueron colectadas en el macrozoobentos durante todo este estudio. Las taxas más dominantes durante este estudio fueron: Melanoides tuberculata (Müller) (Mesogastropoda: Thiaridae) con 3276 individuos (59,36\%), Chironomus sp. (Diptera: Chironomidae) con 1281 individuos $(23,21 \%)$ y Heleobia cumingi (Orbigny) (Mesogastropoda: Hydrobiidae) con 891 individuos (16,14\%). Estas tres especies presentaron una distribución espacial amontonada a lo largo de todos los muestreos realizados. Los valores de diversidad alfa mediante los Indices de diversidad de Shannon-Wiener, de Equidad de Pielou y de Simpson no variaron significativamente entre los siete muestreos realizados y entre las ocho estaciones censadas.
\end{abstract}

Palabras claves: Chironomus, Heleobia, humedales, macrozoobentos, Melanoides, Perú

\begin{abstract}
A study was performed to evaluate the biodiversity and similarity of benthic macroinvertebrates of the Puerto Viejo Lagoons from 15 july to 15 october, 2001. Seven samplings (Sa) were conducted in eight spatial stations $(\mathrm{St})$ in their natural and artificial lagoons. The alpha diversity was calculated using the Shannon-Wiener $\left(\mathrm{H}^{\prime}\right)$, evenness of Pielou and dominance of Simpson (C) indexes. Beta diversity was determined employing quantitative Sörensen and Morisita-Horn indexes. A total of 5519 specimens belonging to three phyla, six orders, nine families and ten species were collected from macrozoobenthos during all the study. The most abundant taxa were Melanoides tuberculata (Müller) (Mesogastropoda: Thiaridae) with 3276 individuals (59,36 \%), Chironomus sp. (Diptera: Chironomidae) with 1281 individuals (23,21 \%) and Heleobia cumingii (Orbigny) (Mesogastropoda: Hydrobiidae) with 891 specimens (16,14\%). These three species had a crowded spatial distribution in all the samplings. The values of alpha diversity: the Shannon-Wiener, equableness of Pielou and Simpson indexes did not change significantly among the seven samplings and in the eight surveyed stations. The values of beta diversity (quantitative indexes of Sörensen and Morisita- Horn) were higher between Sa3 and SA4; and also in $\mathrm{Sa} 5, \mathrm{Sa} 6$ and $\mathrm{Sa} 7$ between each other. For the eight spatial stations Sörensen and Morisita- Horn cuantitative indexes showed a similarity over $70 \%$ between St3- St4; St4- St6 and St5- St7. St2- St6 showed the highest disssimilarity employing both cuantitative indexes.
\end{abstract}

Key words: Chironomus, Heleobia, macrozoobentos, Melanoides, Peru, wetlands.

\section{Introducción}

Los humedales comprenden diversos cuerpos de agua, temporales o permanentes, ámbitos que son hábitats para microorganismos, flora $\mathrm{y}$ fauna características (Brown et al., 1997; Ugarte, 1998; Franco et al., 2000; Pulido 2000). Sin embargo, a pesar que se ha logrado algunos avances encaminados a la conservación de estos ecosistemas sobre todo en la costa, son los primeros que sufren presión antropogénica (Obando et al., 1998), por lo que aún queda mucho por hacer en el campo legal (CONAM,
1999), político y económico (Reaño \& Guardia, 2001).

Las comunidades bentónicas sean marinas $\mathrm{o}$ epicontinentales son todas las especies que viven en relación íntima con el fondo, ya sea para fijarse en él, para excavarlo, o para movilizarse sobre su superficie, siendo el factor básico para su establecimiento, "su relación íntima con el fondo" (Salazar et al., 2001). Las especies bentónicas mayores de $1 \mathrm{~mm}$ de longitud, se consideran macrozoobentos (Jaramillo et al., 1998).

\footnotetext{
${ }^{1}$ Laboratorio de Ecofisiología. Facultad de Ciencias Naturales y Matemáticas. Universidad Nacional Federico Villarreal. Calle San Marcos 383, Pueblo Libre, Lima 21, Perú. Correo electrónico: joseiannacone@hotmail.com

2 Jaime Mansilla Correo electrónico:jaime-bmc@yahoo.cm

${ }^{3}$ Karen Ventura Correo electrónico:kavenza98@hotmail.com
} 
La determinación cualitativa y cuantitativa de la comunidad de invertebrados nos sirven como indicadores biológicos de la calidad del agua (Prat et al., 1999; Cauchie et al., 2000; Gaete et al., 2000); así como su rol como transportadores mecánicos de bacterias u otros patógenos por vía externa e interna (Angeler et al., 2000; Kim et al., 2000).

En el departamento de Lima, Perú, se encuentran los humedales de las lagunas de Puerto Viejo, un conjunto de cuerpos de agua con altas salinidades de diferentes profundidades en medio de un extenso gramadal y juncal, que recoge las afloraciones hídricas del acuífero del Río Mala, con un área de doscientas has. Estas lagunas, conforman junto a otros humedales de la Costa, una red de afloramientos casi continuos que son una fuente de vida para muchísimas especies (Reaño \& Guardia, 2001; Pulido 2000). A la fecha existen muy escasos trabajos publicados sobre este ecosistema natural con relación a inventarios y censos de su biota (Ciriaco et al., 1997; León, 1997).

Los estudios sobre lagunas costeras naturales son de urgente necesidad (Arias \& Sierra, 2001), porque se sabe que la destrucción de los hábitats y la extinción de la vida silvestre ocasiona la ruptura de una cadena ecológica (Obando et al., 1998), es por ello el interés de realizar el presente trabajo durante el 2001. La importancia del estudio de este humedal de Puerto Viejo radica en que sirve de refugio, nidificación y alimentación de aves continentales y locales, provenientes del Hemisferio Norte y de zonas altoandinas respectivamente. Muchas de estas aves residentes o migratorias se alimentan del macrozoobentos de estos humedales.

El objetivo de presente trabajo fue evaluar la biodiversidad alfa y beta del macrozoobentos de las Lagunas de Puerto Viejo durante el 15 de Julio al 15 de Octubre del 2001.

\section{Materiales y métodos}

Los pantanos de Puerto Viejo, se localizan en la provincia de Cañete en el distrito de San Antonio de Mala (Km. 71 de la panamericana Sur) (12 33-34' Lat. Sur $76^{\circ} 42-43^{\prime}$ Long. Oeste). En estas lagunas recorren las afloraciones hídricas del acuífero de Mala que están ubicadas paralelamente al océano, sus profundidades oscilan entre $0,3 \mathrm{~m}$ a $2,4 \mathrm{~m}$ y se encuentran a $20 \mathrm{msnm}$, la temperatura fluctúa de 17 y $22{ }^{\circ} \mathrm{C}$. Las plantas vasculares dominantes de éste ecosistema son Distichlis spicata (L.) Green (Poaceae), Salicornia fruticosa, Cyperus laevigatus L. (Cyperaceae), el junquillo Scirpus americanus (Cyperaceae), Potamogeton striatus R \& P. (Potamogetonaceae) y Bacopa monnieri (L.) Pennell (Scrophulariaceae) (León et al., 1995; Reaño \& Guardia, 2001).

Los siete muestreos se realizaron quincenalmente desde el 15 de julio al 15 de octubre de 2001. Se establecieron 8 estaciones de muestreo en las diferentes lagunas (Figura 1). Las características de las ocho estaciones se presentan en Tabla 1.

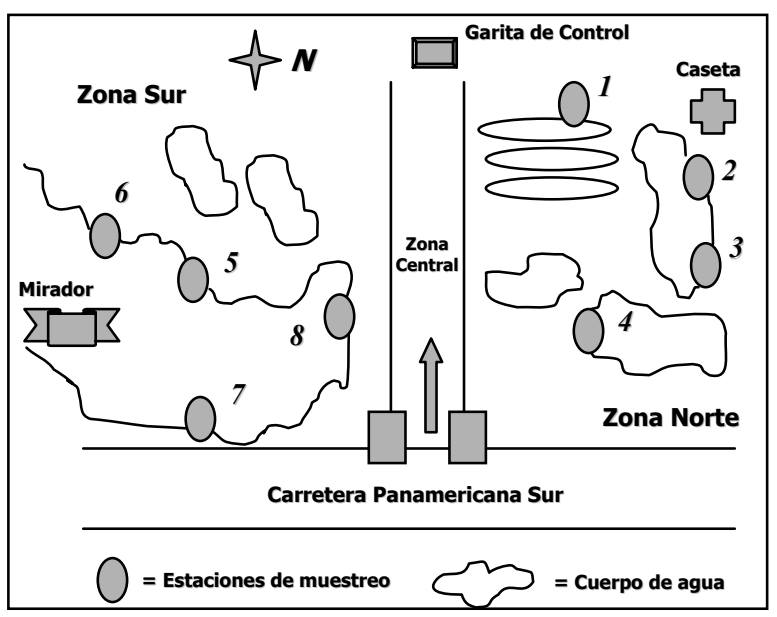

Figura 1. Puntos de muestreo de macrozoobentos en las Lagunas de Puerto Viejo.

Se colectó $1 \mathrm{~L}$ de sustrato bentónico de cada estación a $1 \mathrm{~m}$ de la orilla empleando un envase de plástico de 2 L de capacidad, el cual fue introducido manualmente al sustrato y posteriormente fueron tamizadas con un colador de plástico de $1000 \mu$, para separar el macrozoobentos, siguiendo las recomendaciones de Wetzel \& Likens (2000). Se determinó la temperatura $\left(\mathrm{T}^{\circ}\right), \mathrm{pH}$ y Oxígeno disuelto (estos tres parámetros fueron tomados en todas las estaciones solo en los dos últimos muestreos), presencia de vegetación vascular acuática, tipo de gránulo del sustrato predominante $y$ rango de profundidad de cada estación (Tabla 1). La conductividad eléctrica para todas las estaciones de muestreo fue de $1 \mathrm{mS}$. Además mediante la transparencia del agua se calificó a cada estación en dos categorías: clara o turbia, si es que se observaba o no el disco de Secchi a $10 \mathrm{~cm}$ de profundidad.

En el laboratorio, cada muestra fue separada en morfoespecies y se fijaron en viales de vidrio de $5 \mathrm{~mL}$, con alcohol etílico al $70 \%$, debidamente rotulados para su posterior identificación y clasificación. Para los moluscos se siguió el procedimiento descrito por Thiengo et al. (2001) y para la identificación de los invertebrados se usó claves taxonómicas especializadas (Merritt \& Cummins, 1984; Borror et al., 1995; Vivar et al., 1998; Mazzucconi, 2000). Muestras de importancia taxonómica fueron depositadas en la colección malacológica del Museo de Historia Natural- Universidad Nacional Mayor de San Marcos (MHN, UNMSM) y en la colección entomológica del Programa Nacional de Control Biológico, Servicio Nacional de Sanidad Agraria (PNCB, SENASA), Lima, Perú. Se catalogaron las morfoespecies en tres gremios alimentarios: depredadores, fitófagos y detritívoros empleando los 
criterios propuestos por Iannacone et al. (2000a) y Salazar \& Iannacone (2001).

Con la finalidad de evaluar la diversidad alfa en las comunidades macrozoobentónicas de cada estación y muestreo, fueron utilizados los siguientes índices: Shannon- Wiener, que varía de 0 a log base 2 del número de morfoespecies censadas, determinadas por el número de morfoespecies presentes en la comunidad y basándose en la escala logarítmica escogida $H^{\prime}=-\Sigma \mathrm{p}_{\mathrm{i}} \log \mathrm{p}_{\mathrm{i}}$, donde, $\mathrm{H}^{\prime}=$ índice de diversidad de Shannon-Wiener, $(\log )=$ logaritmo base $2 ; \mathrm{p}_{\mathrm{i}}=\mathrm{n}_{\mathrm{i}} / \mathrm{N}$; donde $\mathrm{n}_{\mathrm{i}}=$ al número de individuos de cada morfoespecie, $\mathrm{N}=$ número total de individuos (Iannacone et al., 2000a). El otro índice utilizado fue el de Simpson (C) que varía entre 0 y 1, cuya fórmula es: $\mathrm{C}=1-\Sigma\left(\mathrm{n}_{\mathrm{i}} / \mathrm{N}\right)^{2}$, donde $\mathrm{n}_{\mathrm{i}}=$ al número de individuos en la muestra o estación, siendo el valor equivalente a 1 como el de máxima diversidad. Se calculó el índice de equidad de Pielou, empleando $\mathrm{J}^{\prime}=$ $\mathrm{H}^{\prime} / \log _{2} \mathrm{~S}$, donde $\mathrm{S}=$ es el número máximo de especies en la muestra. Además, se utilizaron dos procedimientos no paramétricos para estimar la riqueza de especies de este ecosistema: Chao 2 y Jacknife de primer orden (Jack 1) (Moreno, 2001). morfoespecie en el sitio $\mathrm{B}$ (muestreo o estación), da = $\Sigma\left(\mathrm{an}_{\mathrm{i}}\right)^{2} / \mathrm{aN}^{2} \mathrm{y} \mathrm{db}=\Sigma\left(\mathrm{bn}_{\mathrm{j}}\right)^{2} / \mathrm{bN}^{2}$ (Moreno, 2001).

Se empleó el coeficiente de correlación de Pearson para determinar si existía algún tipo de correlación lineal entre las poblaciones de los tres macroinvertebrados más abundantes tanto por muestreo como por estaciones. El ANDEVA se utilizó para determinar si el número de individuos promedio entre los macroinvertebrados más abundantes, $\mathrm{H}^{\prime}, \mathrm{J}$ y C por muestreo y estación por separado, fueron estadísticamente iguales. Para las especies más abundantes se determinó la distribución espacial (al azar, amontonada o uniforme), utilizando el criterio de $\mathrm{S}^{2} / \mathrm{X}$ (Varianza/ Promedio de individuos por estación), realizándose la determinación en cada uno de los siete muestreos. Solo se incluyeron en el análisis las estaciones que presentaron a la especie evaluada. Para todos los casos se empleó un nivel de significancia de alfa $=0,05$.

\section{Resultados}

Se colectaron un total de 5519 ejemplares distribuidos en 10 especies, 9 familias en 6 órdenes. El número total de familias y el número de individuos

Tabla 1. Principales características de las ocho estaciones de los humedales de Puerto Viejo, Lima, Perú.

\begin{tabular}{|c|c|c|c|c|c|c|c|c|}
\hline $\begin{array}{l}\text { Estación } \\
\text { (E) }\end{array}$ & $\begin{array}{l}\text { Tipo } \\
\text { de } \\
\text { laguna }\end{array}$ & $\begin{array}{l}\text { Tipo de } \\
\text { agua }\end{array}$ & $\begin{array}{l}\text { Profundidad } \\
\text { a } 1 \mathrm{~m} \text { de la } \\
\text { orilla (Rango } \\
\text { en } \mathrm{cm} \text { ) }\end{array}$ & $\begin{array}{l}\text { Sustrato } \\
\text { Predominante } \\
\text { del fondo }\end{array}$ & Vegetación & $\begin{array}{l}\text { Temperatura } \\
\mathrm{T}^{\circ} \mathrm{C}\end{array}$ & $\mathrm{PH}$ & $\mathrm{O}_{2}$ disuelto \\
\hline E1 & $\mathrm{A}$ & Clara & $52-78$ & Arenoso & Sí & 23 & 8 & 10 \\
\hline E2 & $\mathrm{N}$ & Clara & $37-54$ & Arcilloso & Sí & 23 & 8 & 8 \\
\hline E3 & $\mathrm{N}$ & Turbia & $15-28$ & Arenoso & Sí & 26 & 8 & 3 \\
\hline E4 & $\mathrm{N}$ & Clara & $40-57$ & Arenoso & No & 25 & 9 & 2 \\
\hline E5 & $\mathrm{N}$ & Clara & $20-45$ & Arenoso & No & 24 & 9 & 3 \\
\hline E6 & A & Turbia & $19-43$ & Arenoso & No & 24 & 9 & 3 \\
\hline E7 & $\mathrm{N}$ & Clara & $2-30$ & Arenoso & Sí & 24 & 9 & 3 \\
\hline E8 & A & Clara & $30-45$ & Arenoso & No & 23 & 8 & 1 \\
\hline
\end{tabular}

$\mathrm{A}=$ Artificial. $\quad \mathrm{N}=$ Natural.

$\mathrm{V}=$ Vegetación = Sí (incluye Cyperus laevigatus, Scirpus americanus, Potamogeton striatus, Distichlis spicata, Bacopa monnieri).

Para la diversidad beta de similaridad, se uso el índice de Sörensen cuantitativo $\left(\mathrm{I}_{\text {Scuant }}\right)=2 \mathrm{pn} / \mathrm{an}+\mathrm{bn}$, donde, an $=$ número total de individuos en el sitio a (muestreo o estación), bn = número total de individuos en el sitio b (muestreo o estación), pn = sumatoria de la abundancia mas baja de cada una de las morfoespecies compartidas entre ambos sitios; y el índice de Morisita -Horn, que varía de 0 a un valor máximo próximo a uno. $\quad \mathrm{I}_{\mathrm{M}-\mathrm{H}}=2 \Sigma\left(\mathrm{an}_{\mathrm{i}} \mathrm{xbn}_{\mathrm{j}}\right) /$ $(\mathrm{da}+\mathrm{db}) \mathrm{aNxbN}$, donde: $\mathrm{an}_{\mathrm{i}}=$ número de individuos de la i-ésima morfoespecie en el sitio A (muestreo o estación), $b n_{\mathrm{j}}=$ número de individuos de la $-\mathrm{j}$ ésima colectados en cada uno de los 7 muestreos y 8 estaciones realizados durante los tres meses de monitoreo, se observa en la Tabla 2. El orden Mesogastropoda con dos especies representó el 75,77 $\%$ de toda la muestra censada. La familia con mayor número de especímenes fue Thiaridae, con la especie Melanoides tuberculata (Muller). Las larvas de la familia Chironomidae con Chironomus sp. fue entre los insectos la más predominante (Tabla 2). La familia Hydrobiidae con Heleobia cumingii (Orbigny) con 891 individuos fue el tercer invertebrado en orden de importancia. Con relación a la posición trófica, el gremio alimentario cualitativamente dominante fueron depredadores $(60 \%$ ), luego los detritívoros con $20 \%$ 
y los fitófagos con $20 \%$; en cambio cuantitativamente, los fitófagos dominaron con 75,5 $\%$, luego los detritívoros con 23,34 \% y finalmente con un menor porcentaje estuvieron los depredadores $(1,12 \%)$ (Tabla 2$)$. cumingii $=127,28 \pm 122,06(0-257)(\mathrm{F}=3,45 ; \mathrm{g} .1$. $=2$ y $18 ; \mathrm{P}=0,05)$.

La Figura 3 nos indica la variación del número de individuos totales de M. tuberculata, Chironomus sp. y $H$. cumingii con relación a las ocho estaciones realizadas en los humedales de Puerto Viejo. Sin

Tabla 2. Macrozoobentos de la Laguna de Puerto Viejo, Lima, Perú durante el 15 de julio Al 15 de octubre del 2001.

\begin{tabular}{|c|c|c|c|c|}
\hline Orden & Familia & Especie & Posición Trófica & $\begin{array}{l}\text { Número } \\
\text { (Abundancia } \\
\text { porcentual) }\end{array}$ \\
\hline Mesogastropoda & Thiaridae & Melanoides tuberculata & $\mathrm{F}$ & $3276(59,36)$ \\
\hline Diptera & Chironomidae & Chironomus sp. (l) & $\mathrm{D}$ & $1281(23,21)$ \\
\hline Mesogastropoda & Hydrobiidae & Heleobia cumingii & $\mathrm{F}$ & $891(16,14)$ \\
\hline Hemiptera & Notonectidae* & Notonecta peruviana $(a, n i)$ & $\mathrm{P}$ & $30(0,54)$ \\
\hline Odonata & Coenagrionidae & sp. no identificada ( $n a)$ & $\mathrm{P}$ & $24(0,43)$ \\
\hline Diptera & Culicidae* & Aedes sp. $(l, p)$ & $\mathrm{D}$ & $10(0,18)$ \\
\hline Coleoptera & Hydrophilidae & Berosus sp. (l) & $\mathrm{P}$ & $3(0,06)$ \\
\hline Tricladida & Planariidae & Girardia festai & $\mathrm{P}$ & $2(0,04)$ \\
\hline Odonata & Libellulidae & sp. no identificada ( $n a$ ) & $\mathrm{P}$ & $1(0,02)$ \\
\hline Coleoptera & Hydrophilidae & Tropisternus sp. (l) & $\mathrm{P}$ & $1(0,02)$ \\
\hline $\mathrm{P}=$ Depredador & $\mathrm{D}=$ Detritívoro & $\mathrm{F}=$ Fitófago & & \\
\hline
\end{tabular}

embargo, el análisis de correlación lineal mostró que solo existe relación negativa y significativa entre

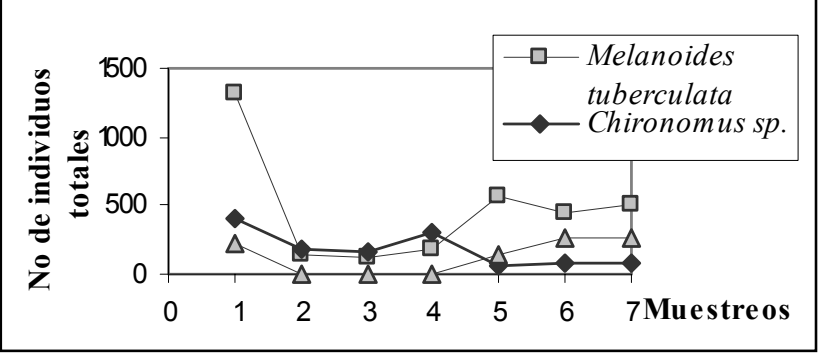
las poblaciones de Chironomus y Heleobia (Tabla 3). Además el ANDEVA mostró que el número promedio de individuos por estación para estas tres especies no varió significativamente entre sí $(M$. tuberculata $=409,50 \pm 548,05(0-1421)$; Chironomus sp. $=160,12 \pm 35,87(95-202)$ y $H$. cumingii $=111,37 \pm 95,89(5-294) ; \mathrm{F}=1,97$; g. 1 . $=2$ y $21 ; \mathrm{P}=0,16)$.

Figura 2. Variaciones poblacionales de tres macroinvertebrados en las Lagunas de Puerto Viejo, Lima, Perú.

La Figura 2 nos indica la variación del número de individuos totales de M. tuberculata, Chironomus sp. y $H$. cumingii con relación a los siete muestreos realizados en los humedales de Puerto Viejo. Se puede observar una tendencia a disminución del número total de larvas de III y IV estadio de Chironomus a través de los muestreos realizados; en cambio Heleobia, a excepción del primer muestreo presenta una tendencia a incrementar sus poblaciones. Sin embargo, el análisis de correlación lineal mostró que no existe ningún tipo de relación significativa entre las poblaciones de estos tres invertebrados por muestreo (Tabla 3). Además el ANDEVA mostró que el número promedio de individuos por muestreo para estas tres especies varió significativamente entre sí (M. tuberculata $=468 \pm 413,73(127-1311)$; Chironomus sp. $=183 \pm 130,64(52-409)$ y $H$.

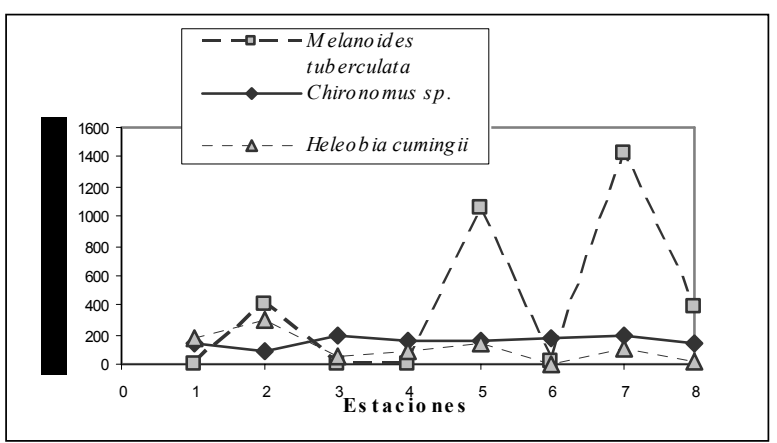

Figura 3. Variaciones poblacionales espaciales de tres macroinvertebrados en las Lagunas de Puerto Viejo, Lima, Perú.

Para casi todos los casos la distribución espacial de M. tuberculata, Chironomus sp. y $H$. cumingii fue amontonada, debido a que presentaron valores mayores a 1 a la relación Varianza/ Promedio del número de individuos (Tabla 4). Solo en el segundo 
muestreo para $M$. tuberculata se observó una distribución al azar, quizás influenciado por ser solo dos estaciones las que presentaron este caracol.

Tabla 3. Matriz de correlación entre el número de individuos totales, según fecha de muestreo y según estación de muestreo, para tres especies de macroinvertebrados de las Lagunas de Puerto Viejo, Lima, Perú.

\begin{tabular}{|c|c|c|c|c|}
\hline & & Por Muestr & & \\
\hline & & $\begin{array}{l}\text { Melanoides } \\
\text { tuberculata }\end{array}$ & $\begin{array}{c}\text { Chironomus } \\
\text { sp. }\end{array}$ & $\begin{array}{l}\text { Heleobia } \\
\text { cumingii }\end{array}$ \\
\hline & Melanoides & - & $0,46^{*}$ & 0,67 \\
\hline & tuberculata & & $(0,29)^{* *}$ & $(0,09)$ \\
\hline$\therefore$ & Chironomus & 0,15 & - & $-0,20$ \\
\hline$\frac{\pi}{\omega}$ & & $(0,71)$ & & $(0,66)$ \\
\hline II & Heleobia & 0,17 & $\underline{-0,71}$ & - \\
\hline 2 & cumingii & $(0,67)$ & $(0,048)$ & \\
\hline & Coeficiente d & e correlación & de Pearson & \\
\hline$* *$ & $=$ Significanci & a o significaci & ón observada & (valor P) \\
\hline & $\begin{array}{l}\text { res sobre la } \\
\text { especies seg }\end{array}$ & $\begin{array}{l}\text { iagonal corre } \\
\text { ún fechas de } 1\end{array}$ & $\begin{array}{l}\text { sponden a cor } \\
\text { nuestreo }(\mathrm{n}=\end{array}$ & $\begin{array}{l}\text { elaciones } \\
\text { 7). }\end{array}$ \\
\hline $\mathrm{Va}$ & res debajo de & la diagonal c & orresponden a & \\
\hline $\begin{array}{l}\mathrm{cor} \\
\mathrm{mu}\end{array}$ & $\begin{array}{l}\text { aciones ent } \\
\text { reo }(n=8) \text {. }\end{array}$ & especies s & in estaciones & \\
\hline
\end{tabular}

Tabla 4. Valores de distribución espacial (Varianza / Promedio de individuos) de tres macroinvertebrados de las lagunas de Puerto Viejo, Lima, Perú.

\begin{tabular}{|c|c|c|c|c|c|c|}
\hline 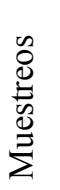 & 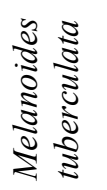 & 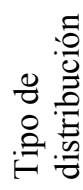 & 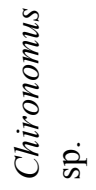 & 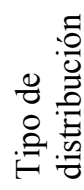 & 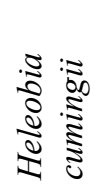 & 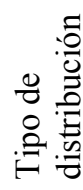 \\
\hline M1 & 436,57 & $A$ & 27,14 & A & 22,77 & A \\
\hline M2 & 0,18 & $\mathrm{Az}$ & 11,80 & A & $-*$ & - \\
\hline M3 & 4,16 & A & 11,20 & A & - & - \\
\hline M4 & 18,36 & A & 14,53 & A & - & - \\
\hline M5 & 36,20 & A & 3,56 & A & 13,09 & A \\
\hline M6 & 23,81 & A & 6,46 & A & 42,61 & A \\
\hline M7 & 52,84 & A & 6,54 & A & 37,04 & A \\
\hline
\end{tabular}

$*=$ se presentó esta especie en $\leq$ a una estación.

$\mathrm{A}=$ distribución amontonada.

$\mathrm{Az}=$ distribución al azar.

Los resultados de los Indices de diversidad de Shannon-Wiener $\left(\mathrm{H}^{\prime}\right)$ y de equidad $\left(\mathrm{J}^{\prime}\right)$, así como el de Simpson (C), calculados para los siete muestreos temporales y para las ocho estaciones espaciales, muestran que el H' con mayor valor promedio fue en el segundo muestreo $(0,99)$ y con el menor valor el cuarto muestreo $(0,69)$. C muestra valores más bajos, siendo el mayor valor el segundo y tercer muestreo $(0,43)$ y con el menor valor en el primer muestreo $(0,35)$. J en el primer muestreo alcanzó el mayor valor
$(0,89)$ y el menor valor lo presentó en el quinto muestreo temporal $(0,49)$. Con relación a las estaciones de muestreo (E), H' presentó el mayor valor promedio en la segunda estación $(0,99)$ y con el menor valor la sexta estación $(0,55)$. C muestra valores más bajos, siendo el mayor valor la tercera estación $(0,49)$ y con el menor valor en la primera estación $(0,26)$. J en la cuarta estación alcanzó el mayor valor $(0,87)$ y el menor valor lo presentó en la sexta estación espacial $(0,49)$. Sin embargo, no se encontraron diferencias significativas en los valores de $\mathrm{H}^{\prime}, \mathrm{J}^{\prime}$ y $\mathrm{C}$, ni por muestreo, ni tampoco por estaciones para los macroinvertebrados de los humedales de Puerto Viejo (Tabla 5).

Tabla 5. Valores comparativos del estadístico F y sus respectivos valores de probabilidad entre $\mathrm{H}^{\prime}, \mathrm{J}^{\prime}$ y $\mathrm{C}$ para los muestreos temporales y estaciones espaciales del macrozoobentos de las Lagunas de Puerto Viejo, Lima, Perú $(\mathrm{F}=$ Prueba de Fisher del ANDEVA; $\mathrm{P}=$ Probabilidad; g.l.= grados de libertad).

\begin{tabular}{|c|c|c|c|c|c|c|}
\hline 导 & 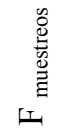 & 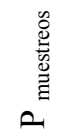 & ¿) & 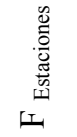 & 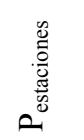 & $\underset{\dot{\infty}}{\dot{0}}$ \\
\hline $\mathbf{H}^{\prime}$ & 0,86 & 0,52 & 5 y 33 & 1,43 & 0,23 & 7 y 31 \\
\hline $\mathbf{J}^{\prime}$ & 0,65 & 0,66 & 5 y 33 & 1,91 & 0,10 & 7 y 31 \\
\hline C & 0,16 & 0,97 & 5 y 33 & 1,21 & 0,33 & 7 y 31 \\
\hline
\end{tabular}

Los dos procedimientos no paramétricos para estimar la riqueza específica de este humedal: Chao 2 y Jacknife de primer orden (Jack 1), para la comunidad de macroinvertebrados de Puerto Viejo, obtuvieron valores de 18 y 13,5 respectivamente. Lo cual indica en comparación a la riqueza de especies de 10, obtenida a lo largo de todo el muestreo, que aún se requiere un mayor monitoreo de los macroinvertebrados bentónicos durante otras épocas del año.

Para evaluar la similaridad por muestreos temporales los índices cuantitativos de Sörensen y Morisita-Horn mostraron coincidencia entre los M3 y M4; así como entre M5, M6, M7 entre sí. Sin embargo, solo Sörensen indicó una alta similaridad entre M2 y M4 (Tabla 6). En contraste, solo Morisita indicó una similaridad entre M1 con M5, M6 y M7. Por ambos índices en los muestreos M1 y M3; así como M1 y M4 presentaron la más alta disimilaridad en todo el monitoreo.

Para las ocho estaciones espaciales, el índice de Sörensen cuantitativo mostró similaridades sobre el 70\% entre E3 y E4; E4 y E6; E5 y E7, y E2 y E8. En cambio para Morisita- Horn E3 y E4; E3 y E6; E4 y E6; E5 y E7; E5 y E8, y entre E7 y E8 presentaron las mayores similaridades. Las estaciones E2 y E6 presentaron la mayor disimilaridad empleando ambos índices cuantitativos (Tabla 7). 
Tabla 6. Matriz de similaridad cuantitativa del macrozoobentos entre los siete muestreos de las Lagunas de Puerto Viejo, Lima, Perú.

\begin{tabular}{|c|c|c|c|c|c|c|c|c|}
\hline & \multicolumn{7}{|c|}{ Sörensen cuantitativo } \\
\hline & & M1 & M2 & M3 & M4 & M5 & M6 & M7 \\
\hline \multirow{7}{*}{ 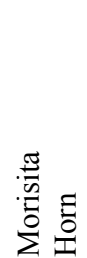 } & M1 & - & 0,282 & 0,264 & 0,397 & 0,506 & 0,539 & 0,589 \\
\hline & M2 & 0,834 & - & 0,958 & 0,738 & 0,339 & 0,399 & 0,392 \\
\hline & M3 & 0,304 & 0,096 & - & $\underline{0,720}$ & 0,316 & 0,377 & 0,375 \\
\hline & M4 & 0,079 & 0,099 & $\underline{0,990}$ & - & 0,375 & 0,413 & 0,406 \\
\hline & M5 & 1,060 & 0,653 & $\overline{0,660}$ & 0,608 & - & 0,794 & 0,842 \\
\hline & M6 & $\overline{1,203}$ & 0,642 & 0,623 & 0,427 & $\underline{0,892}$ & - & $\overline{0,939}$ \\
\hline & M7 & $\overline{0,992}$ & 0,667 & 0,629 & 0,607 & $\overline{0,978}$ & $\underline{0,999}$ & - \\
\hline
\end{tabular}

M1-M7 = Muestras temporales

Índices debajo de la diagonal corresponden a Morisita Horn, índices sobre la diagonal corresponden a Sörensen cuantitativo.

Tabla 7. Matriz de similaridad cuantitativa del macrozoobentos entre las ocho estaciones de las Lagunas de Puerto Viejo, Lima, Perú.

\begin{tabular}{|c|c|c|c|c|c|c|c|c|}
\hline & & & & \multicolumn{5}{|c|}{ Sörensen cuantitativo } \\
\hline & E1 & E2 & E3 & E4 & E5 & E6 & E7 & E8 \\
\hline E1 & - & 0,470 & 0,670 & $\underline{0,940}$ & 0,330 & 0,530 & 0,210 & 0,350 \\
\hline E2 & 0,560 & - & 0,250 & 0,310 & 0,570 & 0,190 & 0,420 & 0,700 \\
\hline E3 & 0,850 & 0,345 & - & 0,780 & 0,220 & 0,670 & 0,170 & 0,390 \\
\hline E4 & $\overline{0,271}$ & 0,416 & $\underline{1,000}$ & - & 0,310 & $\underline{0,730}$ & 0,230 & 0,420 \\
\hline E5 & 0,208 & $\underline{0,860}$ & $\overline{0,189}$ & 0,199 & - & $\overline{0,220}$ & $\underline{0,800}$ & 0,570 \\
\hline E6 & 0,603 & $\overline{0,111}$ & $\underline{0,998}$ & $\underline{0,871}$ & 0,239 & - & $\overline{0,180}$ & 0,460 \\
\hline E7 & 0,171 & $\underline{0,829}$ & $\overline{0,205}$ & $\overline{0,254}$ & $\underline{0,999}$ & 0,279 & - & 0,440 \\
\hline E8 & 0,260 & $\overline{0,818}$ & 0,365 & 0,350 & $\overline{0,976}$ & 0,445 & $\underline{0,982}$ & - \\
\hline
\end{tabular}

$\mathrm{E} 1-\mathrm{E} 8=$ Estaciones espaciales

Índices debajo de la diagonal corresponden a Morisita Horn, índices sobre la diagonal corresponden a Sörensen cuantitativo.

\section{Discusión}

El caracol dulceacuícola $M$. tuberculata es una especie introducida al Perú, pues se cree que su lugar de origen es Malasia (Sudeste Asiático) (Gutierrez et al., 1995). Sin embargo, Vivar et al. (1998) señalan que su localidad tipo es en Timor (Africa). Actualmente se encuentra ampliamente distribuido, inclusive en la región Neártica y en el Neotrópico. Este gasterópodo dioico es de reproducción rápida y partenogénico y con capacidad vivípara u ovovivípara, por ello fácilmente ha colonizado diferentes cuerpos de agua con relativa facilidad, desplazando a otros moluscos, y hallándose en diversas localidades en toda la Costa Peruana (Vivar, 1990). Ha sido registrada en los Humedales de Pantanos de Villa, principalmente en las acequias laterales que desembocan en la laguna principal. También se le ha encontrado en ambientes artificiales en el distrito de La Molina, Lima, Perú (J. Iannacone, observación personal). Aunque se alimentan de microalgas y plantas acuáticas, en algunas ocasiones pudiera comportarse como detritívoro. Además, tiene un alto poder competitivo, al igual que Physa venustula (Gould) (Iannacone \& Alvariño, 1999). Esta alta capacidad de adecuación y dispersión en diferentes tipos de ambientes para $M$. tuberculata ha permitido que se le haya encontrado en todas las estaciones y en los siete muestreos (Tabla 2) (Vivar et al., 1996).

La familia Chironomidae, es uno de los taxas mejor representados en los cuerpos bénticos epicontinentales del Neotrópico (Spies \& Reiss, 1996; Iannacone \& Alvariño, 2000; Fittkau, 2001; Butakka et al., 2002; Moretto et al., 2002; Porto et al., 2002). A nivel mundial, los quironómidos, es una familia comúnmente usada para determinar la toxicidad en sedimentos y la bioacumulación de los contaminantes asociados a estos, la U. S. Environmental Protection Agency (EPA) y la Agency Society for Testing and Materials (ASTM) han publicado procedimientos detallados para el estudio de la toxicidad en sedimentos con Chironomus tentans y Chironomus riparius (Pascoe et al., 2000; Arrascue et al., 2001), siendo ampliamente usados como modelos biológicos, para evaluar los cuerpos de aguas y sedimentos directos y sus elutriados. La especie Chironomus calligraphus Goeldi ha sido estandarizada su crianza y bioensayo, y sido usado para el monitoreo del río Rimac, Lima, Perú y sus niveles de contaminación por metales pesados (Iannacone \& Dale, 1999; Iannacone et al., 2000b), y para evaluar el grado de toxicidad de sedimentos (Iannacone \& Alvariño, 1999; Arrascue et al., 2001). La familia Chironomidae, ha sido predominante en este estudio, al igual que en el humedal costero de las Albuferas de Medio Mundo, Lima, Perú (Iannacone et al., 2001).

Vivar et al. (1998) para los humedales de Los Pantanos de Villa, señalan que $H$. cumingii es una de las especies malacológicas más abundantes, capaz de soportar ambientes contaminados, aunque sus poblaciones son capaces de fluctuar en los diferentes sitios de muestreo por acción de la dureza total, incremento de materia orgánica, concentración de cloruros y la profundidad. En contraste, en nuestro estudio, un número sumamente bajo de especímenes de $H$. cumingii fueron colectados de la E6, quizás debido a la turbidez del agua (Tabla 1; Figura 3).

Iannacone et al. (2000c) para la zona de los Humedales de Ventanilla, en la Provincia constitucional del Callao, Perú registró 2 taxas componentes de los invertebrados de esta laguna costera, Aedes taeniorhynchus (Culicidae) y Notonectidae. Estas dos familias han sido también registradas en el presente estudio (Tabla 2). 
Melo et al. (2002) y Santos et al. (2002) han evaluado la fauna de coleópteros acuáticos en el neotrópico de Brazil, registrando a las larvas de la familia Hydrophilidae, con Tropisternus y Berosus como los géneros más abundantes, dentro de los coleópteros. En el presente estudio también se registró a ambos géneros en las Lagunas costeras de Puerto Viejo, Lima, Perú.

El turbelario Girardia festai (= Dugesia festai) registrado para el humedal de Pantanos de Villa, se encuentran presente solo en la séptima estación de muestreo (Sarmiento \& Morales, 1998). No han sido registrados en Puerto Viejo, moscas dipteras ciclorrafas muy comunes en humedales dulceacuícolas (Keiper et al., 2000).

Como señaló Vivar et al. (1998) el estudio de los macroinvertebrados de Los Pantanos de Villa, Lima, Perú constituyen un grupo de especies claves para el monitoreo y conservación de ese ecosistema particular, pues esta biota acuática, puede actuar como hospederos intermediarios, controladores biológicos, indicadores de la calidad del agua y parte del flujo energético para algunos vertebrados.

Algunos muestreos temporales son más similares entre sí, como es el caso de M5, M6 y M7; así como entre M3 y M4 (Tabla 6). Los parámetros ecológicos evaluados en la Tabla 1, durante el periodo de estudio de 90 días, no fueron muy fluctuantes. Por lo que se requeriría mayores estudios para determinar si la insolación, salinidad u otros factores fisicoquímicos serían los responsables de explicar las altas similaridades encontradas en dichos muestreos.

Las estaciones de muestreo que mostraron las mayores consistencias por ambos índices de similaridad fueron E3 y E4; E4 y E6, y finalmente E5 y E7 (Tabla 7). E3 y E4 fueron bastante similares debido a que fueron lagunas naturales, presentaron sustrato arenoso, temperaturas semejantes, pero ligeramente más altas que el resto de las estaciones y oxígeno disuelto similares (Tabla 1). Así como la cercanía espacial de ambos puntos de muestreo (Figura 1). E4 y E6 son coincidentes en la ausencia de vegetación, fondo arenoso, temperatura, $\mathrm{pH}$ y oxígeno disuelto (Tabla 1). Finalmente para E5 y E7 su similaridad se relaciona a que ambas son lagunas naturales, presentan aguas claras, sustrato arenoso, temperatura, $\mathrm{pH}$ y oxígeno disuelto (Tabla 1). Así como la cercanía espacial de ambos puntos de muestreo (Figura 1).

Los gremios de fitófagos y detritívoros incluyeron pocas especies con un número altos de especímenes con amplia distribución espacial y temporal. En contraste los depredadores fueron numéricamente en bajo número, pero con mayor riqueza de taxas (Tabla 2). Este mismo patrón fue encontrado en la fauna de invertebrados en los humedales de Ventanilla, Callao, Perú (Iannacone et al., 2000b).
El censo de la fauna de invertebrados en el presente estudio para la Laguna de Puerto Viejo, humedal como Los Pantanos de Villa, permite también justificar el establecimiento de estrategias para la conservación de este ecosistema. Observaciones adicionales deberían ser hechas en estos cuerpos de agua para determinar el cambio espacio -temporal de los componentes de macroinvertebrados bentónicos.

Entre las principales conclusiones de este trabajo tenemos: (1) un total de 5519 especímenes, tres filas, seis órdenes, nueve familias y diez especies fueron colectados en el macrozoobentos durante todo este estudio; (2) las tres especies más dominantes durante este estudio fueron: Melanoides tuberculata (Müller) (Mesogastropoda: Thiaridae) con 3276 individuos (59,36 \%), Chironomus sp. (Diptera: Chironomidae) con 1281 individuos $(23,21 \%)$ y Heleobia cumingii (Mesogastropoda: Hydrobiidae) con 891 individuos $(16,14 \%)$. Estas tres especies presentaron una distribución espacial amontonada o contagiosa; (3) los valores de diversidad alfa: los Indices de diversidad de Shannon-Wiener, de Equidad de Pielou y de Simpson no variaron significativamente entre los siete muestreos realizados y entre las ocho estaciones censadas; (4) los valores de diversidad beta (los índices cuantitativos de Sörensen y Morisita-Horn) por muestreo fueron más altos entre los M3 y M4; así como entre M5, M6, M7 entre sí. Por ambos índices en los muestreos M1 y M3; así como M1 y M4 presentaron la más alta disimilaridad en todo el monitoreo y (5) para las ocho estaciones espaciales, los índices de Sörensen y Morisita- Horn cuantitativo mostraron similaridades sobre el 70\% entre E3 y E4; E4 y E6; E5 y E7. Las estaciones E2 y E6 presentaron la mayor disimilaridad empleando ambos índices cuantitativos.

\section{Agradecimientos}

A María Isabel La Torre Acuy por la identificación taxonómica de la flora vascular de los humedales de Puerto Viejo.

\section{Literatura citada}

Angeler D.G., Alvarez-Cobelas M., Rojo C. \& Sánchez-Carrillo S. 2000. The significance of water inputs to plankton biomass and trophic relationships in a semi-arid freshwater wetland (central Spain). J. Plankton Res. 22: 2075- 2093.

Arias C.N.F. \& Sierra P.C. 2001. La Gestión ambiental marina y costera en Colombia: un reto institucional. Libro de Resúmenes del IX Congreso Latinoamericano sobre Ciencias del Mar. San Andrés Isla, Colombia. Sept. 16 - 20, 2001. Universidad Nacional de Colombia. : 501-504.

Arrascue A., Iannacone J., Alvariño L., Basilio S. \& Lazcano C. 2001. El insecto Chironomus calligraphus Goeldi y la bacteria Escherichia coli 
como ensayos ecotoxicológicos para evaluar sedimentos elutriados dulceacuícolas. Rev. per. Ent. 42: 159-173.

Borror D., De Long D. \& Thriplehorn C. 1995. An introduction to the study of insects. Saunders College Publishing. $6^{\text {th }}$ Edition. USA.

Brown S.C., Smith K. \& Batzer D. 1997. Macroinvertebrate responses to wetland restoration in Northern New York. Environ. Entomol. 26: 1016-1024.

Butakka C.M.M., Komatsu E.H. \& Takeda A.M. 2002. Composição da fauna bentônica da lagoa Ventura, sistema rio Ivinheima, bacia do Alto Rio Parana (MS, PR). XXIV Congresso Brasileiro de Zoologia: A Zoologia e os Ecossistemas Costeiros. 17 a 22 de fevereiro de 2002, Itajaí - Santa Catarina, Brazil. : 256.

Cauchie H.M., Hoffmann L. \& Thomé J.P. 2000. Metazooplankton dynamics and secondary production of Daphnia magna (Crustacea) in an aerated waste stabilization pond. J. Plankton Res. 22: $2263-2287$.

CONAM (Consejo Nacional del Ambiente). 1999. Perú Megadiverso. Prioridades en uso y conservación de la biodiversidad para el desarrollo sostenible. Del Río, M. L. (Ed.). Lima, Perú.

Ciriaco F.J., Campos G., Rodríguez C. \& Aguilar C. 1997. Caracterización del ecosistema lagunas de Puerto Viejo. Libro de Resúmenes del Congreso Peruano de Ecología, 25 al 28 marzo de 1997, Lima-Perú. Universidad Nacional Agraria La Molina. : 53.

Fittkau E.J. 2001. Amazonian Chironomidae (Diptera: Chironomidae): A contribution to chironomid research in the neotropics. Amazoniana. 16: 313324.

Franco P.J., Sulca Q.L. \& Cáceres M.C. 2000. Cyanophytas de los Humedales del Valle de Ite Tacna. Libro de Resúmenes del VIII Congreso Nacional de Botánica, Abril 24- 28, 2000. Arequipa. : 51.

Gaete H., Bay-Schmith E., Baeza J. \& Rodríguez J. 2000. Ecotoxicological assessment of two pulps mill effluent, Biobio River Basin, Chile. Bull. Environ. Contam. Toxicol. 65: 183-189.

Gutierrez A.A., Perera G.P., Yong M.C., Ferrer J.L. \& Sánchez J.N. 1995. Distribución y posible competencia entre Melanoides tuberculata y Tarebia granifera (Prosobranchia: Thiaridae) en el lago Hanabanilla, Cuba. Rev. Cubana Med. Trop. 15: 1-7.

Iannacone J., Alayo M., Arrascue A., Sánchez J. \& Abanto M. 2001. Las trampas de luz para evaluaciones rápidas de la biodiversidad de la artropofauna: análisis de tres casos. Wiñay Yachay. 5: 7-20.

Iannacone J., Alayo M. \& Sánchez J. 2000a. Biodiversidad de la artropofauna del Bosque
Zárate, Lima, Perú, empleando tres técnicas de censo. Wiñay Yachay. 4(1): 27-46.

Iannacone J. \& Alvariño L. 1999. Ecotoxicidad aguda de metales pesados empleando juveniles del caracol de agua dulce Physa venustula (Gould, 1847) (Mollusca). Gayana. 63: 101-110.

Iannacone J. \& Alvariño L. 2000. Chironomus calligraphus Goeldi y Moina macrocopa (Sars) como herramientas ecotoxicológicas para la evaluación del lindano y clorpirifos. Bol. Soc. Biol. Concepción. 71: 33-39.

Iannacone J., Alvariño L., Moreno R., Reyes M. \& Chauca J. 2000b. Culícidos (Diptera) del río Chillón y sectores adyacentes de la provincia constitucional del Callao, Perú, durante el Niño 1997-1998. Acta Ent. Chilena. 24: 51-60.

Iannacone J. \& Dale W. 1999. Protocolo de bioensayos ecotoxicológicos para evaluar metales pesados en el agua dulce con Chironomus calligraphus (Diptera: Chironomidae) y Moina macrocopa (Crustácea: Cladocera), en el río Rímac, Lima, Perú. Rev. per. Ent. 41: 111-120.

Iannacone J., Dale W. \& Alvariño L. 2000c. Monitoreo ecotoxicológico del río Rímac (LimaPerú) empleando a Chironomus calligraphus Goeldi (Diptera: Chironomidae). Rev. Chilena Ent. 27: 23-34.

Jaramillo E., Carrasco F., Quijon P., Pino M. \& Contreras H. 1998. Distribución y estructura comunitaria de la macrofauna bentónica en la costa del norte de Chile. Rev. Chilena Hist. Nat. 71: 459-478.

Keiper J.B., Walton W.E. \& Foote B.A. 2002. Biology and ecology of the higher diptera from freshwater wetlands. Annu. Rev. Entomol. 47: 207-232.

Kim H.W., Hwang S.J. \& Joo G.J. 2000. Zooplankton grazing on bacteria and phytoplankton in a regulated large river (Nakdong River, Korea). J. Plankton Res. 22: 1559 - 1577.

León B. 1997. Uso acertado de los humedales de la Costa Central: Retos y posibilidades. Resúmenes del Congreso Peruano de Ecología, 25 al 28 marzo de 1997, Lima-Perú. Universidad Nacional Agraria La Molina. : 6 .

León B., Cano A. \& Young K.R. 1995. La flora vascular de los Pantanos de Villa, Lima, Perú: Adiciones y guía para las especies comunes. Pub. Mus. Hist. Nat., UNMSM (B). 38: 1-39.

Mazzucconi S.A. 2000. A review of the South american Notonecta (Paranecta) including N. inca new species from Peru (Heteroptera: Notonectidae). First Abstract Book I. XXI International Congress of Entomology, Brazil.: 267.

Melo M., Mattos M.L.T., Martins J.F.S., Dias R.A., Rodenas P.A., Benetti C.J. \& Florentín G.L. 2002. Registro de coleoptera em ecossistema de arroz irrigado tratado com inseticida. XXIV Congresso 
Brasileiro de Zoologia: A Zoologia e os Ecossistemas Costeiros. 17 a 22 de fevereiro de 2002, Itajaí - Santa Catarina, Brazil. : 256.

Merrit R.W. \& Cummins K.M. 1984. An introduction to the aquatic insects of North America. Kendall. Hunt Publishing Co. Dubuque, IA. U.S.A.

Moreno C.E. 2001. Métodos para medir la biodiversidad. Manuales y Tesis SEA. Sociedad Entomológica Aragonesa Ed. Madrid, España.

Moretto Y., Takeda A.M. \& Butaca C.M.M. 2002. Distribução espacial de larvas de Chironomidae (Diptera) em seis diferentes ambientes da planície aluvial do Rio Paraná. XXIV Congresso Brasileiro de Zoologia: A Zoologia e os Ecossistemas Costeiros. 17 a 22 de fevereiro de 2002, Itajaí Santa Catarina, Brazil. : 587.

Obando C.M., Campos C.M., García Z.P. \& Romero N.M. 1998. Inventario de la diversidad ornitológica del humedal del caucato Pisco durante 1997. Ecología (Perú). 1: 72-75.

Pascoe D., Wenzel A., Janssen C., Girling A.E., Jüttner I., Fliedner A., Blockwell S.J., Maund S.J., Taylor E.J., Diedrich M., Persoone G., Verhelst P., Stephenson R.R., Crossland N.O., Mitchell G.C., Pearson N., Tatterfield L., Lay J.P., Peither A., Neumeier B. \& Velletti A.R. 2000. The development of toxicity tests for freshwater pollutants and their validation in stream and pond mesocosms. Wat. Res. 34: 2323-2329.

Porto L.A.C., Ribeiro K.D. \& Silva R.C. 2002. Invertebrados bentônicos do alto rio TocantinsGoiás. XXIV Congresso Brasileiro de Zoologia: A Zoologia e os Ecossistemas Costeiros. 17 a 22 de fevereiro de 2002, Itajaí - Santa Catarina, Brazil. : 584.

Prat N., Toja J., Sola C., Burgos M.D., Plans M. \& Rieradevall M. 1999. Effect of dumping and cleaning activities on the aquatic ecosystems of the Guadiamar River following a toxic flood. Sci. Total Environ. 242: 231 - 248.

Pulido V. 2000. Memorias sobre el Taller de Conservación de Humedales de Sitios Ramsar en el Pacífico Sur. 20 -21 de octubre de 1999, Trujillo, Perú.

Reaño G. \& Guardia M. 2001. Salvemos Puerto Viejo. Recurso ecoturístico de Lima. Disponible en: http//:www.conam.gob.pe/chm/docs/otros/eco/otro s/ecoptoviejo leído el 20 de noviembre del 2001.

Salazar N.C. \& Iannacone J. 2001. Censos rápidos empleando la técnica de barber para evaluar la artropofauna del Parque Nacional YanachagaChemillén, Sector Rio Pescado Oxapampa- Pasco. Bol. Lima (Perú). 125: 126-130.
Salazar N.C., Iannacone J., Alvariño L., Orozco R. \& Miranda R. 2001 Estructura macrozoobentónica de fondo blando en la bahía de Ilo, MoqueguaPerú. Wiñay Yachay. 5: 179-192.

Santos A.D.D., Ferreira Jr.N. \& Nessimian J.L. 2002. Caracterização das formas adultas e larvais de Hydrophiloidea (Insecta, Coleoptera) sensu Hansen 1991, para o estado do Rio de Janeiro e localidades vizinhas. XXIV Congresso Brasileiro de Zoologia: A Zoologia e os Ecossistemas Costeiros. 17 a 22 de fevereiro de 2002, Itajaí Santa Catarina, Brazil. : 256.

Sarmiento L. \& Morales M.E. 1998. Protozoarios, turbelarios y nemátodos de los Pantanos de Villa, Lima, Perú: su importancia en el ecosistema. pp. 41-54. Boletín del Museo de Historia Natural, Universidad Nacional Mayor de San Marcos. Serie de divulgación No 11. Cano, A.; Young, K. R. (Eds.). Lima, Perú.

Spies M. \& Reiss F. 1996. Catalog and bibliography of Neotropical and Mexican Chironomidae. Spixiana. 22: 61-119.

Thiengo S.C., Fernández M.A., Boaventura M.F., Grault C.E., Silva H.F.R., Mattos A.C. \& Santos S.B. 2001. Freshwater snails and schistosomiasis mansoni in the state of Rio de Janeiro, Brazil: IMetropolitan Mesoregion. Mem. Inst. Oswaldo Cruz. 96: 177-184.

Ugarte J.A.N. 1998. Los humedales de la Reserva Nacional Salinas y Aguada Blanca: 21000 Flamencos que proteger. Libro de Resúmenes del IV Congreso Latinoamericano de Ecología. II Congreso Peruano de Ecología. 20 al 25 de Octubre 1998. Arequipa- Perú. Instituto Regional de Ciencias Ambientales. Facultad de Ciencias Biológicas y Agropecuarias. Departamento Académico de Biología. Universidad San Agustín. Arequipa - Perú.

Vivar R. 1990. Un gasterópodo de la familia Thiaridae en el Perú: Melanoides tuberculata (Müller, 1774). Bol. Lima (Perú). 69: 33-34.

Vivar R., Larrea H., Huaman P., Yong M. \& Perera G. 1996. Some ecological aspects of the freshwater molluscan fauna of Pantanos de Villa, Lima, Perú. Malacol. Rev. 29: 65- 68.

Vivar G.R., Ramírez M.R. \& Huamán P. 1998. Moluscos de los Pantanos de Villa y su aporte a la Conservación. pp. 55-73. Boletín del Museo de Historia Natural, Universidad Nacional Mayor de San Marcos. Serie de divulgación No 11. Cano, A.; Young, K. R. (Eds.). Lima, Perú.

Wetzel R.G. \& Likens G.E. 2000. Limnological analyses. 3th Ed. Springer- Verlag, New York. 